\title{
Thermovision System Based on the IR CCD Matrix on PtSi with Shottky Barrier
}

\author{
V.D.Ivanov, S.B.Tihomirov \\ “Jupiter-Z”, St.Petersburg, Russia mailto:vdi@peterlink.ru \\ G.A.Agranov, V.K.Nesterov, G.V.Levko, V.O.Timofeev \\ NRI Electron, St.Petersburg, Russia
}

\begin{abstract}
Multipurpose thermovision system based upon the infrared CCD matrix with Shottky barrier has been build. Echnical parameters and software details (automatical contrasting, dynamic averaging) are considered.
\end{abstract}

The second-generation thermovision system is based upon the infrared focal plane array. The focal plane array is monolithic platinum silicide Schottky-barrier IR charge coupled device image sensor. It is designed for infrared imaging applications in the $1 \div 5 \mu \mathrm{m}$ wavelength range.

The device has interline-transfer architecture and is oriented for backside illumination. Thin films of platinum silicide are used in manufacturing of Schottky-barrier detectors. The readout is carried out by vertical and horizontal CCD. Both CCDs have a buried channel, and are driven by four-phase clock pulses. The output preamplifier is a floating diffusion amplifier with a two-stage source follower.

The high thermal and spatial resolution of device, low noise readout, and high uniformity produce a good quality thermal images in real time.

\section{Technical characteristics}

Architecture

Pixels number

Pixel size interline CCD, interlaced $256(\mathrm{H}) \times 290(\mathrm{~V})$

$50 \mu \mathrm{m}(\mathrm{H}) \times 33 \mu \mathrm{m}(\mathrm{V})$ 
Imager size

Aspect ratio

Frame time

Operation temperature

Spectral response (w/o filter)

Integration time

Pixel readout rate

Saturation signal

Electron sensitivity

Dynamic range
$12.8 \mathrm{~mm}(\mathrm{H}) \times 9.57 \mathrm{~mm}(\mathrm{~V})$

$4: 3$

$40 \mathrm{~ms}$

$77 \mathrm{~K}$

$1 \div 5.5 \mu \mathrm{m}$

$37 \mathrm{~ms}$

$5.0 \mathrm{MHz}$

$7 \times 10^{5}$ electrons

$5 \mu \mathrm{V} /$ electron

$60 \mathrm{~dB}$

$\operatorname{NETD}(\mathrm{f} / 1.280 \mathrm{~mm}$ lens $) \leq 0.1 \mathrm{~K}$

\section{Software}

Software is MacOS ${ }^{\text {TM }}$ based and has been tested on the Power Macintosh ${ }^{\text {TM }} 7600,8600$ computers. The choice of a platform is due to the OS-level of video-in support and high performance in the image and video processing.

The image processing includes the indemnification of distortions and defects of the sensor based on the calibration previously recorded, original algorithm of frame accumulation (optional), pseudo-colorization, loss-less visual enhancement, the number of digital filters etc. Frame rate of completely processed, filtered and colorized thermoimages is 25 frame per second. Software provides real-time recording on the disk and playback the movie as well as still images in QuickTime format that is compatible with Windows-95. Such a movie may be played back later and used exactly the same way as real input from the camera to analyze and investigation. Standard image file formats (TIFF, JPEG, QuickTime and others) are supported and may be managed by any image database. As example, template for FileMaker database is included. Software has component structure and compatible with worldwide Adobe PhotoShop ${ }^{\mathrm{TM}}$ plug-ins standard. Thus, a number of third-part filters may be used.

Software package may be explored with any other equipment involving standard videoout (composite or S-Video, NTSC, PAL, SECAM). 
The camera under consideration has low noise level allowing receiving high quality images without accumulation - 25 fps. However, in terms of sensitivity increasing, or with more noisy video source, averaging of frame may be used. Original algorithm of adaptive exponential averaging is provided. Averaging factor is dynamically changed depending on speed of the image variability (object's movement or trend of temperature) and is various for different parts of the image. It helps to increase the SNR for motionless objects and at the same time to observe fast movements without a delay or blur - at the expense of some increase of noise level

A number of digital linear and nonlinear filters, including noise reduction, smoothing, edge detection etc. is provided for image enhancement.

Another approach is proposed to improve a visual representation without changing of data, namely "adaptive color palette". The original algorithm minimizes a root-meansquare error of representation at the expense of dynamic non-uniform change of color levels depending on histogram of intensity. This adaptive color palette is optional applied with pseudo-colorization and may dramatically enhance the contrast. The same algorithm is used for automatic thresholding of "object on the background".

Software tools includes measurement of temperature in any points, temperature profiles, histogram and statistical data on chosen area etc. These tools may be applied with realtime monitoring as well as with still snapshots. Time-dependence results of measurement may be plotted.

\section{Image processing}

The goal of our software development is to let real-time video processing. In common, the software works with live video in the same manner as with still images. All the filters, measurement tools and palette applied to video stream on the fly, so we can see, for example, variation of the temperature profile over the surface while object is heated or cooled. Video could be recorded on the disk and played later. Recorded movie could be played and used exactly as live video.

"Non-invasive processing" paradigm is the other distinctive feature. It means that we tend to keep the source images ("rough data") unchanged and apply filters and 
algorithmes on the fly when playing images or video. The only exclusion is the "matrix correction" procedure which is applied in the video-in driver at the very first step of image acquisition.

Saving on a disk image or video, we write the "rough ", source data. Applied filters, tools and palette are saved separately from data in the same file. So the image could be restored in its original form anytime as well as opened in the latter visual form.

\section{Matrix correction.}

Matrix correction is a kind of calibration that is used to compensate ununiformity of the CCD matrix or to exclude defective elements from the image. We use two-points linear correction based on the labs testing of the camera. The individual correction coefficients calculated for every elements of the matrix. If there are elements which correction coefficients are out of bounds, they should be excluded from the image and replaced by interpolated values. The ununiformity profile of the matrix is very stable and it is not necessary to repeat calibration procedure. In common such a correction applied immediately in the driver at the very first step of capturing. User can disable correction or apply another calibration however - the calibration procedure is very simple. One useful application is to subtract background or exclude other kind of ununiformity, e.g. illumination or filter transparency variation.

\section{Adaptive palette.}

Visual enhancement of the image can be achieved with pseudo-color or contrasting colorisation. Contrast palette is applied on the fly when image sent to the screen. Modern computer has powerful facilities for that. In common, colorisation palette can be described as transform from the data (pixel) space to some color space:

$R=P * X$,

where $\boldsymbol{X}$ - available data values set (e.g. $(0,4096)$ for the 12-bit digitizer), $\boldsymbol{R}$ - color space (8-bit grayscale, RGB, HSV etc), $\boldsymbol{P}$ - "palette", optimal in some meaning nonlinear (in common) operator. If operator $\boldsymbol{P}$ depends on the global characteristic of the image (e.g. on the luminance histogram), we call such a palette "adaptive". 
There are a lot of optimisation criterion and corresponding algorithms in the literature. Let us get as a basic the classic problem of the optimal quantization [1], which is based on the intuitive understanding of the optimal criterion. Divide the diapason $X$ by the $\boldsymbol{L}$ intervals and replace values in the $k$ interval by the some value $z_{k}$. Define square-root error estimation as:

$$
E=\sum_{k=1}^{L} \int_{z_{k}}^{z_{k+1}}\left(z-q_{k}\right)^{2} h(z) d z
$$

The optimal quantization problem is then reformulated as the minimization of the objective function $\boldsymbol{E}$. Zeroing the derivatives with respect to $q_{k}$ and $z_{k}$ we obtain:

$$
\begin{gathered}
z_{k}=\frac{q_{k-1}+q_{k}}{2} \\
q_{k}=\frac{\int_{z_{k}}^{z_{k+1}} z h(z) d z}{\int_{z_{k}}^{z_{k+1}} h(z) d z}
\end{gathered}
$$

The equations can be solved by simple iteration algorithm, because, in fact, it is a recursive formula to calculate $q_{k}$ and $z_{k}$.

Note that in the case $L=2$ equations (2) describe widely used binarization algorithm $[2,3,4]$. This binarization method sometimes called LSTSM - Least Square Threshold Selection Method.

However, as shown in [5], even in case of binarization $(L=2)$ objective function (1) can be multimodal and have many local minimumes. Surely in most cases there's no single minimum when $L>2$.

In order to achieve absolute minimum of (1) we had to use straight global minimization of the objective function. Histogram function has not so many points (256-4096) so it is not the time-consuming operation. In calculations histogram function was smoothed by interpolation in fractional values. Finally it has been shown that the better results achieved when small values of $\boldsymbol{L}(\sim 8)$ used and $(256 / \boldsymbol{L})$ levels of luminosity are uniformly distributed in the intervals $\left(q_{k}, q_{k-1}\right)$, 
Such a half-empirical algorithm has been proved as very fast and effective in visual enhancement. Note that it is a completely automatical and therefore can be used in the "one push button" technology (user gets the visual enhancement by one push of the button).

Fig. 1,2,3 illustrated applying grayscale and pseudocolor adaptive palette to IR image.

\section{Dynamic averaging.}

Frame averaging is widely used method to increase signal to noise relation (SNR). When summarized $\mathrm{N}$ frames signal amplitude growth as $N$ and gaussian noise as $\sqrt{N}$. More than, averaging can effectively increase dynamic width of the signal (number of the significant bits) if summarized with extra bits to avoid overfloating or loosing the least significant bits.

While it works fine with stationary scene, the moving objects are blurred. In fact the frame averaging can be expressed as FIR (finite impulse responce) filter which degradates the time-scale resolution. More than, smooth windowing with aperture $\mathrm{W}$ and applied FIR filter requires to store $\mathrm{W}$ frames and, therefore, is a very resource-hungry procedure. Anyway in order to increase SNR in 5 times we have to average 25 frames $(\mathrm{W}=25)$ and therefore we'll get about 1 frame per second (fps). It is almost impractical for dynamic images, moving scene or live video.

The simple but effective decision is to use IIR (infinite impulse responce), or recursive filter with coefficients varyed depending on the local variability of the image. Local $1^{\text {st }}$ order IIR filter can be written as

$p_{t}(x, y)=\alpha \cdot q_{t}(x, y)+(1-\alpha) \cdot p_{t-1}(x, y)$,

where $\mathrm{p}$ - averaged pixel value at the time $\boldsymbol{t}$ and $\boldsymbol{t}-\boldsymbol{1}$ corresponding, $\boldsymbol{q}$ - original (captured) pixel value. Such a filter is local because pixel value completely defined by the history of the same pixel.

Another advantage of the IIR filter is that it is enough to store only 2 frames in order to get filter with any aperture. 
The simple idea is to vary coefficient $\alpha$ depending on the variation speed at the same points, or, better, at the some neighbourhood of this point:

$$
\alpha(x, y)=\Phi\left\{\frac{d\left(\int G \cdot q(x, y) d x d y\right)}{d t}\right\},
$$

where $\mathrm{G}$ is the Gaussian-like kernel providing stability of the IIR filter.

Obviously the filter becomes non-linear (with respect to time) but still can be considered as local (with respect to coordinates) even with $\alpha$ depending on the pixel's neighbourhood. We call such a filtering "dynamic averaging" due to the fact that it is a general method of averaging suitable for non-stationary scene. Functional $\Phi$ should be empirically selected (we used polynomial representation). With appropriated parameters of (4) reasonable balance between SNR increasing and blurring can be achieved. Visual enchancement will be even better because we get low noise level at the stationary regions whereas higher noise at the rapidly changed scene is not so noticeable.

This method has been used with IR as well as with other kind of technical videos and reasonable results has been achieved even with $\mathrm{SNR}<0.1$

\section{Literature}

1. Max J., Quantizing for Minimum Distortion, IRE Transactions on Information Theory, IT-6, 7-12 (1960)

2. Otsu N. - In: Proc. $4^{\text {th }}$ Int. Joint Conf. On Pattern Recognition, p.592-596 (1978)

3. Otsu N. - IEEE Trans., v. SMC-9, No.1, p.62-66 (1979)

4. Trussel H.J. - IEEE Trans., v.SMC-9, No.5, p.311 (1979)

5. Kittler J., Illingworth J., Foglein J. - Computer Vision, Graphics and Image Processing, v.30, No.2, p.125-147 (1985) 
http://dx.doi.org/10.21611/qirt.2000.054 